\title{
Use of platelet-rich plasma in the treatment of infertility in poor responders in assisted human reproduction procedures
}

\author{
IRINA PACU ${ }^{1,2}$, NIKOLAOS ZYGOUROPOULOS ${ }^{2}$, MIHAI DIMITRIU ${ }^{1,2}$, \\ GEORGE ROSU $^{1,2}$ and CRINGU A. IONESCU ${ }^{1,2}$ \\ ${ }^{1}$ Department of Obstetrics and Gynecology, 'Carol Davila' University of Medicine and Pharmacy, 050474 Bucharest; \\ ${ }^{2}$ Department of Obstetrics and Gynecology, 'Sf. Pantelimon' Emergency Clinical Hospital, 021623 Bucharest, Romania
}

Received August 6, 2021; Accepted September 7, 2021

DOI: $10.3892 /$ etm.2021.10848

\begin{abstract}
Couple infertility is a pathology with an absolute number of cases growing markedly over the last decade in connection mainly with the increased age of couples wishing to conceive. Platelet-rich plasma (PRP) is an alternative treatment used for several years for experimental purposes. Yet, this method is not yet defined as a standard therapeutic option in the infertility protocol for poor responders in assisted human reproduction procedures. Thus, the present study is a retrospective study conducted between February 2019 and February 2020 to evaluate the effect of ovarian PRP injection in patients with a poor ovarian response (POR) to ovarian stimulation. Women ( $\mathrm{n}=20$; age $31-44$ years) diagnosed with POR based on the European Society of Human Reproduction and Embryology criteria underwent ovarian injection with autologous PRP injection. Markers of ovarian reserve before, during the following two menstrual cycles, and at six months after treatment were followed as well as stimulation and fertilisation parameters before and post-treatment. PRP treatment resulted in increased antral follicle count and serum anti-Mullerian hormone, while levels of serum follicle-stimulating hormone and luteinising hormone were decreased. These changes were
\end{abstract}

Correspondence to: Dr Irina Pacu, Department of Obstetrics and Gynecology, 'Sf. Pantelimon' Emergency Clinical Hospital, 340-342 Pantelimon Boulevard, 021623 Bucharest, Romania

E-mail: irinapacu@hotmail.com

Abbreviations: AFC, number of antral follicles; $\mathrm{AMH}$, anti-Mullerian hormone; ARC, assisted reproduction cycle; ESHRE, European Society of Human Reproduction and Embryology; ET, embryo-transfer; FSH, follicular stimulating hormone; GH, growth hormone; GnRH, gonadotropin-releasing hormone; HSG, human chorionic gonadotropin; ICSI, intracytoplasmic sperm injection; LH, luteinising hormone; MI, meiosis I; MII, metaphase II; PRP, platelet-rich plasma; POR, poor ovarian response; POSEIDON, Patient-Oriented Strategies Encompassing Individuals Oocyte Number; VEGF, vascular endothelial growth factor

Key words: platelet-rich plasma, poor ovarian response, in vitro fertilisation, assisted reproduction cycle, anti-Mullerian hormone more pronounced during the 2nd menstrual cycle following treatment. By six months following the injection, their values return to pre-treatment levels and any small differences were not considered statistically significant. The average dose of gonadotropin used and duration remained statistically unchanged, but a significant increase in estradiol achieved by the day of the human chorionic gonadotropin trigger day was achieved. The cancellation rate decreased following PRP treatment while the number of collected oocytes, number of oocytes in metaphase II rose. The number of embryos (of A and B quality) resulting also increased but fell short of the significance level set $(\alpha=0.073)$. Following the PRP injection, two singleton pregnancies were achieved, resulting in live births at term without complications during pregnancy. Another pregnancy was achieved spontaneously 45 months following the PRP and a failed assisted human reproduction procedure. Although the group included a small number of women, the results indicate the potential benefits of an ovarian autologous PRP injection in women with POR. Positive results appear to be short-term for 2-6 months after the procedure.

\section{Introduction}

Couple infertility is a pathology with an absolute number of cases growing markedly over the last decade in connection mainly with the increased age of couples wishing to conceive, with a significant change in living and working conditions in a modern technological society. Medical progress in the field of infertility research and treatment offers increasing chances for cases that a few years ago seemed unresolved, constantly looking for new therapeutic solutions.

In this context, there is an increasingly common pathology related to anovulatory infertility with various causes relating to genetic factors, behavioral enzymes, autoimmune diseases, increased maternal age, environmental factors, or combinations thereof $(1,2)$. Excluding polycystic ovary syndrome, which is the most common cause of anovulatory female infertility, an important place is occupied by the category of patients with diminished ovarian reserve, which includes those with a poor ovarian response (POR) (1). The definition of this category has been closely related to the response to ovarian stimulation in assisted human reproduction procedures which are the only therapeutic option indicated for these cases. 
Initially, the Bologna criteria were defined (ESHRE 2011), which classifies POR patients as those who meet at least two of the following criteria: age over 40 years, poor response to previous procedures (cycle canceled or less than 3 oocytes collected), decreased ovarian reserve number of antral follicles (AFC) below 5-7, and anti-Mullerian hormone (AMH) $<1.2 \mathrm{ng} / \mathrm{ml}$ ). An additional two episodes of POR are sufficient for classification regardless of the criteria $(3,4)$.

Subsequently, in clinical practice, there is the problem of defining criteria that would include a wider range of aspects and a more precise stratification, allowing standardised therapeutic conduct for each category. In this sense, the POSEIDON (Patient-Oriented Strategies Encompassing Individuals Oocyte Number) group has developed a new classification that extends maternal age and no longer considers it as a defining factor, focusing on previous experience of response to ovarian stimulation, AMH, and antral follicle number (5). This is defined as follows:

POSEIDON 1: patients under 35 years of age with normal ovarian reserve but unexpected POR (AMH $\geq 1.2 \mathrm{ng} / \mathrm{ml}$, AFC $\geq 5)$; i) 1a: $<4$ oocytes collected in the previous assisted reproduction cycle (ARC); ii) 1b: 4-9 oocytes collected in the previous ARC.

POSEIDON 2: patients over/or 35 years of age with normal ovarian reserve $(\mathrm{AMH} \geq 1.2 \mathrm{ng} / \mathrm{ml}, \mathrm{AFC} \geq 5)$ but unexpected POR: i) 1a: $<4$ oocytes collected in the previous ARC; ii) 1b: 4-9 oocytes collected in the previous ARC.

POSEIDON 3: patients under 35 years of age with decreased ovarian reserve $(\mathrm{AMH}<1.2 \mathrm{ng} / \mathrm{ml}$, AFC $<5)$.

POSEIDON 4: patients over/or 35 years of age with decreased ovarian reserve (AMH $<1.2 \mathrm{ng} / \mathrm{ml}, \mathrm{AFC}<5)$.

The use of these criteria is closer to the clinical reality that frequently identifies cases with poor response to stimulation in younger ages or with moderately diminished ovarian reserve. Often these cases are accompanied by a low number of poor-quality oocytes and the impossibility of having embryos of good enough quality to obtain a pregnancy.

One benefit of using the POSEIDON classification is that it expands the group of these patients to include a new category of hypo-responders in addition to the classic poor responders $(3,5)$. These are patients who do not generally belong to the age group over 40 years, with moderately diminished ovarian reserve but who have responded very poorly to stimulation with high doses of gonadotropins (6). The number of antral follicles and the value of AMH cannot accurately predict the response to stimulation, and the explanation may be related to increased resistance of gonadotropins in these patients by genetic factors [for example genetic mutations or single nucleotide polymorphisms $(3,7,8)]$. These patients are often included in the category of poor responders; they differ from the classical group and even the use of the POSEIDON classification allows their inclusion only after the clinical experience of two failed assisted human reproduction procedures by lack of quality embryos, very low number of embryos or lack of oocytes in metaphase II (MII). The POSEIDON classification that was also used by us in this study manages to allow a better stratification of these patients which results in improved chances of obtaining a pregnancy (5).

To improve the chances of obtaining a pregnancy in poor responders, various therapeutic strategies and protocols have been tried: alternative gonadotropin preparations and regimens, androgen supplementation (testosterone or dehydroepiandrosterone), use of androgen-modulating agents (letrozole), the use of growth hormone $(\mathrm{GH})$, luteinising hormone $(\mathrm{LH})$ pretreatment, and antioxidants, without the possibility of finding an 'ideal' recipe $(7,9,10)$.

Platelet-rich plasma (PRP) is an alternative treatment used for several years for experimental purposes. The idea for its use started from clinical experience in other fields of medicine (dermatology, orthopedics, plastic surgery) and consists of obtaining a platelet concentrate obtained from plasma collected from the patient in question by centrifuging the peripheral blood collected $(11,12)$. This platelet concentrate brings with it over 700 proteins, hormones, growth factors, immunomodulators, and hormones and proteins with biological activity $(3,13)$. These factors will stimulate angiogenesis, anabolic processes, cell migration, cell differentiation, and proliferation in the tissues where they are injected [autograft procedure $(14,15)]$. The present study starts from the promising results of other studies with a statistically significant number of cases related to PRP injection in the ovaries of poor responders, in patients with premature ovarian failure or menopause, studies with experimental value but whose results certify the benefits of this therapeutic options (16). The method is not yet defined as a standard therapeutic option in the infertility protocol in these categories of patients (3).

\section{Patients and methods}

The present study is a retrospective study conducted between February 2019 and February 2020 at the 'St. Pantelimon' Emergency Hospital in Bucharest and the Genesis Athens Bucharest Assisted Human Reproduction Clinic to evaluate the effect of ovarian PRP injection in patients with POR. A total of 20 patients were selected with a mean age of 37.4 and a range of 31 to 44 years. Patients provided a signed informed consent regarding the experimental treatment. They had the ability to freely withdraw from the study without prejudice at any time. Further thay agreed in writing for the use, storage and manipulation of the data collected and agreed for all data to be published anonymously.

Inclusion and exclusion criteria. The inclusion criteria followed the definition for POR patients according to the POSEIDON classification. In all cases, the use of PRP was performed after at least one human assisted reproduction procedure without success, and after PRP injection, in all cases, this was followed by at least one human assisted reproduction procedure.

Exclusion criteria were: an association with male infertility, endocrine dysfunction, autoimmune diseases, thrombophilia, malignancies, infectious diseases, and a family history of neoplastic diseases.

Experimental protocol. The PRP injection was performed in all cases at least 2 months after the last ovarian stimulation procedure. All patients signed informed consent to accept the use of this procedure.

The stimulation protocol used was similar, as in the previous cycle, with the administration of gonadotropins from day 2 of the menstrual cycle [follicular-stimulating hormone 
(FSH) recombinant in combination with urinary $\mathrm{FSH}$, with the basal determination of plasma estradiol on that day before starting stimulation. The initiation of stimulation took place only in patients whose estradiol level was below $50 \mathrm{pg} / \mathrm{ml}$. Pituitary inhibition was performed with $0.25 \mathrm{mg}$ gonadotropin-releasing hormone $(\mathrm{GnRH})$ antagonist per day when the largest ovarian follicle was at least $14 \mathrm{~mm}$, and oocyte maturation was achieved by administering $250 \mathrm{mg}$ human chorionic gonadotropin (HCG) 30-34 h before the follicular puncture at an average follicular diameter of $18 \mathrm{~mm}$. The follicles were aspirated with a negative pressure of 1,120-1,250 mmHg using a single-lumen needle $17-\mathrm{G}$ under transvaginal ultrasound guidance.

In all cases, the harvested oocytes were analysed, and intracytoplasmic sperm injection (ICSI) was performed for all oocytes. Luteal phase support was performed in all cases with oral or intravaginal progesterone preparations until plasma HCG dosing was performed. Embryo-transfer (ET) was performed using fresh embryos or cryopreserved depending on the number and quality of embryos obtained and the desire of the patients. The maximum number of embryos transferred was 2.

Data analysis. Data were collected and compared from all patients regarding $\mathrm{AMH}, \mathrm{FSH}$, and $\mathrm{LH}$ values on day 3 of a cycle before, as well as one, two, and six months after the PRP injection procedure. The assisted reproduction procedures were performed at a minimum of 2 months following the PRP injection, in the optimal interval considered 2 to 4 months. $\mathrm{AMH}, \mathrm{FSH}$, and $\mathrm{LH}$ values were assessed on day 3 and 6 months after injection.

We compared data on the doses of gonadotropins used for ovarian stimulation, estradiol value on the day of HCG administration, the number of MII oocytes collected, and the number of good quality embryos obtained in a cycle preceding PRP injection and following the PRP injection.

Embryo quality was assessed on day 2 after ICSI using criteria related to cell division rate, the symmetry of blastomeres and their number, percentage of fragmentation, presence, or absence of multinucleated blastomeres (17).

Assessing the number of oocytes in MII is important for the success of obtaining good quality embryos and subsequently, a pregnancy because although using ICSI can theoretically fertilize all oocytes, only those in MII will give rise to embryos that can lead to pregnancy (18). After denudation, one can identify aspects related to abnormalities of the zona pellucida, abnormal cytoplasmic granularity, smooth endoplasmic clusters, denatured or abnormal polar globules, abnormal perivitelline spaces; all these morphological aspects point to obtaining non-viable embryos (19).

The oocytes in meiosis I (MI) are immature and attempts to mature them in vitro in the first $24 \mathrm{~h}$, meets with success only in $25-45 \%$ of cases. Of these, the fertilization rate is low $(51 \%)$ and the quality of the embryos is poor. After prolonged cultures, most authors report an aneuploidy rate of $40-100 \%(18,19)$

PRP injection. Injection of the PRP concentrate was performed during the early follicular phase, between days 3-5 of the menstrual cycle $(3,20)$. The PRP preparation was prepared in all cases on the day of injection by venous blood collection from a forearm vein, in most cases the medial vein of the forearm (20). The amount of blood collected was $60-80 \mathrm{ml}$ depending on the technical specifications of the collection kit used (EasyPRP kit; Neotec Biotechnology Ltd.) The platelet concentration obtained was 250,000-850,000 platelets/ $\mu 1$.

The injection was performed in two patients during a laparoscopic intervention for diagnostic purposes during the infertility investigation and in 18 patients by an ultrasound-guided transvaginal ovarian puncture. The puncture was performed in all cases under general intravenous anesthesia. The injection was performed with 2-4 ml PRP at the level of the ovarian parenchyma, the approach of the ovary being at a distance from the vascular pedicle to avoid hemorrhagic accidents $(3,20)$. There were no complications related to ovarian puncture in any case.

The cases were analyzed before PRP injection by dosing on day 3 of the menstrual cycle of FSH, LH, AMH, and ultrasound determination of the AFC. The same determinations were made in the first two cycles after the procedure and at an interval of 6 months. We discussed changes in serological parameters 1-2 months after the procedure and then at 6 months to evaluate the benefits of this therapeutic option and the time interval at which we can relate with these possible benefits. We compared the number of oocytes, the number of MII oocytes collected, the number of embryos, and their quality before and after PRP injection.

Statistical analysis. Statistical analysis was performed according to the distribution of the values, employing parametric tests regarding normally distributed values, and non-parametric tests regarding values lacking normal distribution.

Considering the limited number of participants included in the study group $(n=20)$, the Kolmogorov-Smirnov normality test was employed to assess whether the tested data originated from a normally distributed population. In the case of a non-normal distribution, the non-parametric Friedman test was performed and Nemenyi post-hoc analysis to compare the paired outcomes prior and following PRP treatment. For normally distributed values, the parametric paired Student's t-test with unequal variances was employed. Confidence intervals of $95 \%(\alpha=0.05)$ were calculated for each variable, and a P-value $<0.05$ was considered to be statistically significant.

\section{Results and Discussion}

The category of patients with diminished ovarian reserve, regardless of etiology, and who are not always of older age, faces the huge problem of a small cohort of primary follicles. This aspect seems to be related to a reduction in ovarian vascularization, with PRP bringing angiogenic factors that can improve the pathophysiological phenomena (20). Many studies show the benefits of PRP in reducing inflammatory phenomena, reducing intraoperative and postoperative bleeding, reducing infections, and stimulating osteogenesis. Most studies show that vascular endothelial growth factor (VEGF) is involved in these processes $(14,15,20)$. The soluble factors present in PRP favor the intraovarian environment that stimulates the growth of preantral follicles that will have a better response 
Table I. Ovarian reserve markers before and after PRP treatment.

Following PRP injection

\begin{tabular}{lccrr} 
& Before PRP & & & \\
\cline { 3 - 5 } Ovarian reserve markers & Day 3 & 1st menstrual cycle & 2nd menstrual cycle & At 6 months \\
\hline FSH $(\mathrm{UI} / \mathrm{ml})$ & $11.50 \pm 4.05$ & $8.30 \pm 2.13^{\mathrm{a}}$ & $7.05 \pm 1.43^{\mathrm{b}}$ & $11.28 \pm 3.23$ \\
LH $(\mathrm{UI} / \mathrm{ml})$ & $7.25 \pm 1.92$ & $5.10 \pm 1.29^{\mathrm{a}}$ & $5.20 \pm 1.44^{\mathrm{a}}$ & $6.00 \pm 2.36$ \\
AMH $(\mathrm{ng} / \mathrm{ml})$ & $0.69 \pm 0.32$ & $0.82 \pm 0.33$ & $0.99 \pm 0.36^{\mathrm{a}}$ & $0.71 \pm 0.33$ \\
AFC & $3.45 \pm 1.15$ & $4.85 \pm 1.73^{\mathrm{a}}$ & $5.65 \pm 1.81^{\mathrm{b}}$ & $3.85 \pm 1.35$ \\
\hline
\end{tabular}

Data are represented as mean $\pm \mathrm{SD}$. ${ }^{\mathrm{a}} \mathrm{P}<0.05$, significant difference in comparison to day 3 pre-treatment; ${ }^{\mathrm{b}} \mathrm{P}<0.001$, significant difference in comparison to day 3 pre-treatment. PRP, platelet-rich plasma; FSH, follicular-stimulating hormone; LH, luteinising hormone; AMH, anti-Mullerian hormone; AFC, antral follicular count.

Table II. Cycle performance indicators before and after PRP treatment.

\begin{tabular}{lcc}
\hline Cycle performance indicators & Before PRP & Following PRP \\
\hline Duration of stimulation (days) & $10.1 \pm 2.47$ & $10.0 \pm 1.56$ \\
Gonadotropin dose (IU) & $4,002.50 \pm 856.93$ & $3,801.50 \pm 521.68$ \\
Estradiol (pg/ml) (HCG trigger) & $603.75 \pm 262.24$ & $907.75 \pm 386.56^{\mathrm{b}}$ \\
Cancellation rate $^{\mathrm{a}}$ & $40.0 \%(8 / 20)$ & $15.0 \%(3 / 20)$ \\
Retrieved oocytes $^{\mathrm{a}}$ & $0.80 \pm 0.83$ & $1.8 \pm 1.15$ \\
MII oocytes obtained $^{\mathrm{a}}$ & $0.75 \pm 0.62$ & $1.51 \pm 0.87$ \\
Good quality embryos (A and B) $^{\mathrm{a}}$ & $0.33 \pm 0.49$ & $0.76 \pm 0.75$ \\
\hline
\end{tabular}

${ }^{\mathrm{a} C a l c u l a t e d ~ w i t h o u t ~ t a k i n g ~ i n t o ~ a c c o u n t ~ t h e ~ c a n c e l l e d ~ c a s e s . ~ D a t a ~ a r e ~ r e p r e s e n t e d ~ a s ~ m e a n ~} \pm \mathrm{SD}$ or frequency $(\%)$. ${ }^{\mathrm{b}} \mathrm{P}<0.05$, significant difference in comparison to before PRP treatment. PRP, platelet-rich plasma; HCG, human chorionic gonadotropin; MII, metaphase II.

to stimulation with exogenous FSH. However, with advancing age, errors appear at the level of DNA synthesis and therefore of cell division, with the rate of aneuploidies and congenital anomalies being increased (18). For this reason, the time factor is essential in finding therapeutic options for these patients for whom often the only way to get pregnant is by using donated oocytes.

In the present study, the mean age of the patients was $37.4 \pm 4.00$ years. The average and standard deviation of the ovarian reserve markers found before and after treatment are provided in Table I. Before PRP treatment in comparison to the following 1st menstrual cycle, LH, FSH, and AFC significantly improved $(\mathrm{P}<0.05)$ with the exception for $\mathrm{AMH}$, which despite improvement, the difference was not statistically significant. In comparison between before PRP treatment and the 2nd menstrual cycle, all markers (FSH, LH, AMH and AFC) were significantly improved $(\mathrm{P}<0.05)$, in particular FSH and $\mathrm{AFC}(\mathrm{P}<0.001)$. At six months after treatment, despite the mean values remaining lower than before treatment, the differences were not significant. FSH (UI/ml) appeared to register a larger decrease during the 2 nd menstrual cycle $(7.05 \pm 1.43)$ rather than the 1 st cycle $(8.30 \pm 2.13)$ where the significant difference reached $\mathrm{P}<0.001$ in comparison to the level before PRP treatment $(11.50 \pm 4.05)$. Nonetheless, by 6 months post-treatment, the value $(11.28 \pm 3.23)$ returns to similar levels as prior to treatment. $\mathrm{LH}(\mathrm{UI} / \mathrm{ml})$ followed a similar trend to FSH although the difference between the 1st and

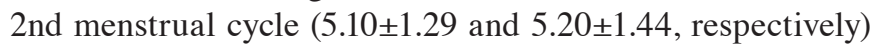
and subsequent recovery to the values before PRP treatment were less prominent (before PRP, $7.25 \pm 1.92$ and at 6 months, $6.00 \pm 2.36)$.

The AMH level (ng/ml) was increased following PRP treatment in both the 1 st $(0.82 \pm 0.33)$ and 2 nd menstrual cycle $(0.99 \pm 0.36)$ reaching a significant increase $(\mathrm{P}<0.05)$ by the 2nd cycle. By the 6 th month following treatment $(0.71 \pm 0.33)$, its value was significantly decreased $(\mathrm{P}<0.05)$ compared to the level at the 2nd menstrual cycle post-treatment, and despite remaining slightly higher than pre-PRP treatment $(0.69 \pm 0.32)$ the difference was not considered significant.

AFC followed a similar trend as AMH with its value increasing significantly from pre-PRP treatment $(3.45 \pm 1.15)$ to the 1 st $(4.85 \pm 1.73)(\mathrm{P}<0.05)$ and more so to the 2 nd menstrual cycle $(5.65 \pm 1.81)(\mathrm{P}<0.001)$ but by the 6 th-month post-treatment $(3.85 \pm 1.35)$, any significant effect had subsided.

Concerning the in vitro fertilisation/ICSI cycles before and after PRP treatment, the average value and standard deviation of the data collected are shown in Table II. The average dose of gonadotropin used was lower following PRP treatment $(3,801.50 \pm 521.68$ vs. $4,002.50 \pm 856.93)$, but the same number of days were required on average. Neither difference was significant. On the other hand, an increase of approximately $50 \%$ in the amount of estradiol achieved by the day of the HCG 
trigger day ( $907.75 \pm 386.56$ vs. $603.75 \pm 262.24)$ was achieved and considered statistically significant with a $\mathrm{P}<0.001$.

The cancellation rate was significantly decreased following PRP treatment from $40 \%(8 / 20)$ to $15 \%$ (3/20). Concurrently, the number of collected oocytes was significantly increased to more than double $(1.8 \pm 1.15$ vs. $0.80 \pm 0.83)$ as well as the number of oocytes that reached MII ( $1.51 \pm 0.87$ vs. $0.75 \pm 0.62)$. Unfortunately, despite the increase in the number of embryos (of A and B quality) $(0.76 \pm 0.75$ vs. $0.33 \pm 0.49)$, the statistical analysis indicated that this increase fell short of the significance level set $(\alpha=0.073)$.

It should be noted that the procedures performed after PRP injection resulted in two singleton pregnancies obtained in the study group, resulting in live births of female fetuses born at term and without complications during pregnancy. In one case, a spontaneous pregnancy was obtained naturally 45 months after PRP and a failed assisted human reproduction procedure.

In conclusion, in the study group, PRP had significant benefits for increasing FSH, LH, and estradiol levels but it was less efficient for increasing AMH. At 6-month intervals, the values returned to levels similar to those preceding the PRP procedure. The number of canceled cycles was significantly reduced. A significantly increased number of MII oocytes and good-quality embryos were obtained. After injection of PRP by assisted human reproduction procedures, two pregnancies were obtained and in one case a pregnancy was obtained naturally.

Although the group included a small number of cases, the results indicate the benefit of ovarian PRP injection for POR. Positive results appear to be short-term for a period of 2-6 months after the procedure. No adverse reagents or complications of the procedure were recorded, so although the method remains an alternative and experimental one, it can be considered as a therapeutic option for POR patients before initiating a new assisted human reproduction procedure after correct information and patient consent. The main factor that negatively impacts the oocyte quality rate of embryos and the pregnancy rate is advanced age, thus this option may have the best results in the POR that did not fall into this category. An important aspect that should be studied further is related to the amount/concentration of PRP vs. the effect, as well as the possibility of repeated injections in several cycles. The limitations of the study are related to a relatively small number of cases and the impossibility of establishing clinical, serological, or ultrasound criteria with which to identify the group of patients with maximum benefits from the use of this method; thus requiring future research.

\section{Acknowledgements}

Not applicable.

\section{Funding}

No funding was received.

\section{Availability of data and materials}

All data (with exception of the actual experimental data) and information was collected through open or paid databases containing published journals. Experimental raw data collected during the study are available on request in accordance to the provisions stipulated in the patient consent for publication.

\section{Authors' contributions}

IP, NZ, MD, GR and CAI were equal contributors in collecting the available data and information. IP and NZ composed the final manuscript. IP in addition coordinated the entire effort and performed the medical procedures. All authors read and approved the final manuscript for publication.

\section{Ethics approval and consent to participate}

Patients provided a signed informed consent regarding the experimental treatment. They had the ability to freely withdraw from the study without prejudice at any time. Further thay agreed in writing for the use, storage and manipulation of the data collected and agreed for all data to be published anonymously. The study and subsequent publication were approved by the Ethics Committee of 'St. Pantelimon' Emergency Hospital in Bucharest (approval no. 19).

\section{Patient consent for publication}

Not applicable.

\section{Competing interests}

The authors declare that they have no competing interests that would prejudice the impartiality of the research reported.

\section{Authors' information}

ORCID numbers: Irina Pacu: 0000-0002-4253-6810; Nikolaos Zygouropoulos: 0000-0002-8404-6158; Mihai Dimitriu: 0000-0002-7454-7389; George Rosu: 0000-0003-4876-5347; Cringu A. Ionescu: 0000-0003-4533-6766.

\section{References}

1. Vollenhoven B and Hunt S: Ovarian ageing and the impact on female fertility. F1000 Res 7: F1000 Faculty Rev-1835, 2018.

2. Lew R: Natural history of ovarian function including assessment of ovarian reserve and premature ovarian failure. Best Pract Res Clin Obstet Gynaecol 55: 2-13, 2019.

3. Sfakianoudis K, Simopoulou M, Grigoriadis S, Pantou A, Tsioulou P, Maziotis E, Rapani A, Giannelou P, Nitsos N, Kokkali G, et al: Reactivating ovarian function through autologous platelet-rich plasma intraovarian infusion: Pilot data on premature ovarian insufficiency, perimenopausal, menopausal, and poor responder women. J Clin Med 9: 1809, 2020.

4. Ferraretti AP, La Marca A, Fauser BC, Tarlatzis B, Nargund $G$ and Gianaroli L; ESHRE working group on poor ovarian response definition: ESHRE consensus on the definition of 'poor response' to ovarian stimulation for in vitro fertilization: The Bologna criteria. Hum Reprod 26: 1616-1624, 2011.

5. Humaidan P, Alviggi C, Fischer R and Esteves SC: The novel POSEIDON stratification of 'Low prognosis patients in Assisted Reproductive Technology' and its proposed marker of successful outcome. F1000Res 5: 2911, 2016.

6. Winship AL, Stringer JM, Liew SH and Hutt KJ: The importance of DNA repair for maintaining oocyte quality in response to anti-cancer treatments, environmental toxins and maternal ageing. Hum Reprod Update 24: 119-134, 2018. 
7. Roman R, Mussarat N and Detti L: Ovarian stimulation in poor responders: Have We Made Progress? Curr Pharm Biotechnol 18: 614-618, 2017.

8. Dimitriu M, Stoian AP, Rosu G, Dan A, Caimacan A Zygouropoulos N, Pacu I, Socea B,Ionescu C, Gheorghiu DC, et al: Postpartum depression-a serious, dangerous, disabling, extremely frequent condition that is almost completely ignored in Romania. Mediterr J Clin Psychol 8: 1-24, 2020.

9. Regan SLP, Knight PG, Yovich JL, Arfuso F and Dharmarajan A: Growth hormone during in vitro fertilization in older women modulates the density of receptors in granulosa cells, with improved pregnancy outcomes. Fertil Steril 110: 1298-1310, 2018.

10. Mihalas BP, Redgrove KA, McLaughlin EA and Nixon B: Molecular mechanisms responsible for increased vulnerability of the ageing oocyte to oxidative damage. Oxid Med Cell Longev 2017: 4015874, 2017.

11. Foster TE, Puskas BL, Mandelbaum BR, Gerhardt MB and Rodeo SA: Platelet-rich plasma: From basic science to clinical applications. Am J Sports Med 37: 2259-2272, 2009.

12. Schmitz JP and Hollinger JO: The biology of platelet-rich plasma. J Oral Maxillofac Surg 59: 1119-1121, 2001.

13. Hosseini L, Shirazi A, Naderi MM, Shams-Esfandabadi N Borjian Boroujeni S, Sarvari A, Sadeghnia S, Behzadi B and Akhondi MM: Platelet-rich plasma promotes the development of isolated human primordial and primary follicles to the preantral stage. Reprod Biomed Online 35: 343-350, 2017.
14. Peterson JE, Zurakowski D, Italiano JE Jr, Michel LV, Fox L, Klement GL and Folkman J: Normal ranges of angiogenesis regulatory proteins in human platelets. Am J Hematol 85: 487-493, 2010.

15. Dawood AS and Salem HA: Current clinical applications of platelet-rich plasma in various gynecological disorders: An appraisal of theory and practice. Clin Exp Reprod Med 45: 67-74, 2018.

16. Marques LF, Stessuk T, Camargo IC, Sabeh Junior N, dos Santos L and Ribeiro-Paes JT: Platelet-rich plasma (PRP): Methodological aspects and clinical applications. Platelets 26: 101-113, 2015.

17. Tesarik J, Junca AM, Hazout A, Aubriot FX, Nathan C, Cohen-Bacrie P and Dumont-Hassan M: Embryos with high implantation potential after intracytoplasmic sperm injection can be recognized by a simple, non-invasive examination of pronuclear morphology. Hum Reprod 15: 1396-1399, 2000.

18. Yu EJ, Ahn H, Lee JM, Jee BC and Kim SH: Fertilization and embryo quality of mature oocytes with specific morphological abnormalities. Clin Exp Reprod Med 42: 156-162, 2015.

19. Rienzi L, Ubaldi FM, Iacobelli M, Minasi MG, Romano S, Ferrero S, Sapienza F, Baroni E, Litwicka K and Greco E: Significance of metaphase II human oocyte morphology on ICSI outcome. Fertil Steril 90: 1692-1700, 2008.

20. Cakiroglu Y, Saltik A, Yuceturk A, Karaosmanoglu O, Kopuk SY, Scott RT Jr, Tiras B and Seli E: Effects of intraovarian injection of autologous platelet rich plasma on ovarian reserve and IVF outcome parameters in women with primary ovarian insufficiency. Aging (Albany NY) 12: 10211-10222, 2020. 